\title{
SO(3) RATIONAL MAP SOLITON IN QUANTUM SU(3) SKYRME MODEL
}

\author{
D. Jurčiukonis and E. Norvaišas \\ Institute of Theoretical Physics and Astronomy of Vilnius University, A. Goštauto 12, LT-01108 Vilnius, Lithuania \\ E-mail: darius@itpa.1t, norvaisas@itpa.lt
}

Received 4 September 2008; accepted 4 December 2008

\begin{abstract}
The quantum Skyrme model is considered in noncanonical bases $\mathrm{SU}(3) \supset \mathrm{SO}(3)$ for the state vectors. A rational map ansatz is used to describe the soliton with the topological number greater than one. The canonical quantization of the Lagrangian generates in Hamiltonian five different "moments of inertia" and negative quantum mass corrections, which can stabilize the quantum soliton solution. Explicit expressions of the quantum Lagrangian and the Hamiltonian are derived for this model soliton.
\end{abstract}

Keywords: Skyrme model, skyrmions, topological solitons, rational map

PACS: 03.65.Fd, 12.39.Dc

\section{Introduction}

The Skyrme model was introduced as an effective theory of baryons [1]. Recently the topological soliton solutions, namely the skyrmions, are applied in studies of the quantum Hall effect, Bose-Einstein condensate, and black hole physics. General analytical solutions of the model are unknown even in the classical case, and approximate solitonic solutions are under intensive consideration. For instance, the rational map ansatz [2] is used to describe light nuclei as quantized skyrmions $[3,4]$.

The original Skyrme model was defined for a unitary field $U(\mathbf{x}, t)$ that belongs to a fundamental representation of the $\mathrm{SU}(2)$ group. A semiclassical quantization suggests that the skyrmion rotates as a "rigid body" [5]. The collective coordinates approach separates the variables that depend on the time and spatial coordinates. The structure of the ansatz which depends on spatial coordinates determines the solitonic solutions. A constructive realization of the canonical quantization adds a term in the Hamiltonian which may be interpreted as an effective pion mass term [6]. An extension of the model to the SU(N) group [7] represents a common structure of the Skyrme Lagrangian and explains its wide application.

The aim of this work is to discuss the grouptheoretical aspects of the canonical quantization of the SU(3) Skyrme model in the rational map ansatz ap- proximation with baryon number $B \geq 2$. The ansatz is defined in noncanonical $\mathrm{SU}(3) \supset \mathrm{SO}(3)$ bases as an $\mathrm{SO}(3)$ solitonic solution. The canonical quantization generates five different "moments of inertia". The proposed ansatz can be used to describe light nuclei as special skyrmions.

The paper is organized as follows. Some preliminary definitions are presented in Sec. 2. The quantum Skyrme model is constructed ab initio in the collective coordinates framework for the rational map soliton in Sec. 3. Section 4 contains a summarizing discussion. A few relevant mathematical details and lengthier expressions of the Hamiltonian terms are given in the Appendix.

\section{Noncanonical embedding of the rational map soliton}

The Skyrme model is a Lagrangian density for a unitary field $U(\mathbf{x}, t)$ that belongs to the general representation of the SU(3) group [8]. We consider the unitary field in the fundamental representation $(1,0)$ of the SU(3) group. The chirally symmetric Lagrangian density has the form

$$
\mathcal{L}=-\frac{f_{\pi}^{2}}{4} \operatorname{Tr}\left(\mathbf{R}_{\mu} \mathbf{R}^{\mu}\right)+\frac{1}{32 \mathrm{e}^{2}} \operatorname{Tr}\left(\left[\mathbf{R}_{\mu}, \mathbf{R}_{\nu}\right]\left[\mathbf{R}^{\mu}, \mathbf{R}^{\nu}\right]\right),
$$


where the "right" and "left" chiral currents are defined as

$$
\begin{aligned}
& \mathbf{R}_{\mu}=\left(\partial_{\mu} U\right) U^{\dagger}=\partial_{\mu} \alpha^{i} C_{i}^{(B)}(\alpha)\left\langle\left|J_{(B)}\right|\right\rangle, \\
& \mathbf{L}_{\mu}=U^{\dagger}\left(\partial_{\mu} U\right)=\partial_{\mu} \alpha^{i} C_{i}^{(B)}(\alpha)\left\langle\left|J_{(B)}\right|\right\rangle
\end{aligned}
$$

and have the values on the SU(3) algebra. The $f_{\pi}$ and $\mathrm{e}$ in (1) are phenomenological parameters of the model. Explicit expressions of functions $C_{i}^{(B)}(\alpha)$ and $C_{i}^{(B)}(\alpha)$ depend on the group parametrization $\alpha^{i}$. The noncanonical $\mathrm{SU}(3)$ generators may be expressed in terms of the canonical generators $J_{(Z, I, M)}^{(1,1)}$ defined in [9]:

$$
\begin{aligned}
& J_{(1,1)}=\sqrt{2}\left(J_{\left(\frac{1}{2}, \frac{1}{2}, \frac{1}{2}\right)}^{(1,1)}-J_{\left(-\frac{1}{2}, \frac{1}{2}, \frac{1}{2}\right)}^{(1,1)}\right) \\
& J_{(1,0)}=2 J_{(0,1,0)}^{(1,1)} \\
& J_{(1,-1)}=\sqrt{2}\left(J_{\left(-\frac{1}{2}, \frac{1}{2},-\frac{1}{2}\right)}^{(1,1)}+J_{\left(\frac{1}{2}, \frac{1}{2},-\frac{1}{2}\right)}^{(1,1)}\right) \\
& J_{(2,2)}=-2 J_{(0,1,1)}^{(1,1)} \\
& J_{(2,1)}=-\sqrt{2}\left(J_{\left(\frac{1}{2}, \frac{1}{2}, \frac{1}{2}\right)}^{(1,1)}+J_{\left(-\frac{1}{2}, \frac{1}{2}, \frac{1}{2}\right)}^{(1,1)}\right) \\
& J_{(2,0)}=-2 J_{(0,0,0)}^{(1,1)}, \\
& J_{(2,-1)}=-\sqrt{2}\left(J_{\left(-\frac{1}{2}, \frac{1}{2},-\frac{1}{2}\right)}^{(1,1)}-J_{\left(\frac{1}{2}, \frac{1}{2},-\frac{1}{2}\right)}^{(1,1)}\right) \\
& J_{(2,-2)}=2 J_{(0,1,-1)}^{(1,1)} .
\end{aligned}
$$

The canonical generators satisfy the following commutation relations:

$$
\begin{aligned}
& {\left[J_{\left(L^{\prime}, M^{\prime}\right)}, J_{\left(L^{\prime \prime}, M^{\prime \prime}\right)}\right]=} \\
& -2 \sqrt{3}\left[\begin{array}{ccc}
(1,1) & (1,1) \\
L^{\prime}, M^{\prime} L^{\prime \prime}, M^{\prime \prime} & (1,1)_{a} \\
L, M
\end{array}\right] J_{(L, M)} .
\end{aligned}
$$

The bracketed coefficients on the right-hand side of (5) are the $\mathrm{SU}(3)$ noncanonical isofactor and the $\mathrm{SO}(3)$ Clebsch-Gordan coefficient. The state vectors for the canonical bases $\mathrm{SU}(3) \supset \mathrm{SU}(2)$ and the noncanonical bases $\mathrm{SU}(3) \supset \mathrm{SO}(3)$ are equivalent in fundamen- tal representation. We suggest to use the rational map ansatz in the $\mathrm{SO}(3)$ case as a matrix

$$
\begin{aligned}
& \left(U_{R}\right)_{M, M^{\prime}}=D_{M, M^{\prime}}^{1}(\varkappa)= \\
& \left\{\exp \left[2 \mathrm{i} \hat{n}_{a} J_{(1, a)} F(r)\right]\right\}_{M, M^{\prime}}= \\
& 2 \sin ^{2} F(-1)^{M} \hat{n}_{-M} \hat{n}_{M^{\prime}}+\mathrm{i} \sqrt{2} \sin 2 F\left[\begin{array}{ccc}
1 & 1 & 1 \\
M & u & M^{\prime}
\end{array}\right] \hat{n}_{u} \\
& +\cos 2 F \delta_{M, M^{\prime}},
\end{aligned}
$$

where the unit vector $\hat{\mathbf{n}}$ is defined [2] in terms of a rational complex function $R(z)$ as

$$
\hat{\mathbf{n}}=\frac{1}{1+|R|^{2}}\left\{2 \operatorname{Re}(R), 2 \operatorname{Im}(R), 1-|R|^{2}\right\} .
$$

The triplet $\varkappa$ of Euler angles is defined by $\hat{n}$ and $F(r)$. By differentiation of $\hat{\mathbf{n}}$ we get an expression which is advantageous in the following calculations,

$$
\begin{aligned}
& (-1)^{s}\left(\nabla_{-s} r \hat{n}_{m}\right)\left(\nabla_{s} r \hat{n}_{m^{\prime}}\right)= \\
& \hat{n}_{m} \hat{n}_{m^{\prime}}+\mathcal{I}\left[(-1)^{m} \delta_{-m, m^{\prime}}-\hat{n}_{m} \hat{n}_{m^{\prime}}\right],
\end{aligned}
$$

where the symbol $\mathcal{I}$ denotes the function

$$
\mathcal{I}(\theta, \varphi)=\left(\frac{1+|z|^{2}}{1+|R|^{2}}\left|\frac{\mathrm{d} R}{\mathrm{~d} z}\right|\right)^{2}
$$

that solely depends on the angles $\theta$ and $\varphi$.

The baryon charge density for the rational map skyrmion is expressed as

$$
\begin{aligned}
& \mathcal{B}(r, \theta, \varphi)=\epsilon^{0 k \ell m} \operatorname{Tr}\left(R_{k} R_{\ell} R_{m}\right)= \\
& -\frac{\mathcal{I}(\theta, \varphi)}{2 \pi^{2}} \frac{F^{\prime}(r) \sin ^{2} F}{r^{2}} .
\end{aligned}
$$

Since this expression contains the $\mathcal{I}$ function, there is no need to modify the usual boundary conditions $F(0)=$ $\pi$ and $F(\infty)=0$ to take into account the chiral angle. The normalized baryon charge density and the Lagrangian density for $\mathrm{SO}(3)$ are reduced by a factor of 4 . The integral by the spatial angles of $\mathcal{I}$ can be regarded as the Morse function and is proportional to the baryon number [10]:

$$
\int_{0}^{2 \pi} \mathrm{d} \varphi \int_{0}^{\pi} \mathrm{d} \theta \mathcal{I} \sin \theta=4 \pi B
$$


With the ansatz (7) the Lagrangian density (1) reduces to the classical Skyrme Lagrangian for any baryon number $B$ :

$$
\begin{aligned}
\mathcal{L}_{\mathrm{cl}}(r, \theta, \varphi)= & -\mathcal{M}_{\mathrm{cl}}=-2 f_{\pi}^{2}\left[F^{\prime 2}(r)+\frac{2 \mathcal{I} \sin ^{2} F}{r^{2}}\right] \\
& -\frac{4}{\mathrm{e}^{2}} \frac{\mathcal{I} \sin ^{2} F}{r^{2}}\left[F^{\prime 2}(r)+\frac{\mathcal{I} \sin ^{2} F}{2 r^{2}}\right],
\end{aligned}
$$

which describes the skyrmion mass density.

\section{Canonical quantization of the soliton}

The quantization of the model can be carried out by means of collective coordinates that separate the variables depending on the time and spatial coordinates,

$$
U(\mathbf{r}, q(t))=A U_{R} A^{\dagger}=A(q(t)) U_{R}(\mathbf{r}) A^{\dagger}(q(t)) .
$$

Here eight $\mathrm{SU}(3)$ group parameters $q^{i}(t), i=1, \ldots, 8$ are quantum variables. Thus the Skyrme Lagrangian is considered quantum mechanically $a b$ initio in contrast to the conventional semiclassical quantization of the soliton as a rigid body. The generalized coordinates $q^{i}(t)$ and the corresponding velocities $\dot{q}^{i}(t)$ satisfy the following commutation relations:

$$
\left[\dot{q}^{\alpha}, q^{\beta}\right]=-\mathrm{i} f^{\alpha \beta}(q)
$$

where $f^{\alpha \beta}(q)$ are functions of $q$ only which are determined below. The commutation relation between the velocity component $\dot{q}^{\alpha}$ and an arbitrary function $G(q)$ is given by

$$
\left[\dot{q}^{\alpha}, G(q)\right]=-\mathrm{i} \sum_{r} f^{\alpha \beta}(q) \frac{\partial}{\partial q^{\beta}} G(q) .
$$

We adopt the usual Weyl ordering for the time derivative:

$$
\partial_{0} G(q)=\frac{1}{2}\left\{\dot{q}^{\alpha}, \frac{\partial}{\partial q^{\alpha}} G(q)\right\} .
$$

The curly brackets denote an anticommutator. Due to the Weyl operator ordering no further ordering ambiguity appears in the Lagrangian or the Hamiltonian. The differentiation of the $q$-dependent unitary matrix can be expressed in terms of functions $C_{\alpha}^{\prime(L, M)}$ and the matrix elements of the group generator $J_{(L, M)}$ :

$$
\begin{aligned}
& \frac{\partial}{\partial q^{\alpha}} G_{(A)(B)}^{(\lambda, \mu)}(q)= \\
& C_{\alpha}^{(L, M)}(q) G_{(A)\left(A^{\prime}\right)}^{(\lambda, \mu)}(q)\left\langle\begin{array}{c}
(\lambda, \mu) \\
A^{\prime}
\end{array}\left|J_{(L, M)}\right| \begin{array}{c}
(\lambda, \mu) \\
(B)
\end{array}\right) .
\end{aligned}
$$

The relations (14)-(16) are neglected in semiclassical calculations.

For the purpose of defining the metric tensor in the Lagrangian we use an approximate expression

$$
A^{\dagger} \dot{A} \approx \frac{1}{2}\left\{\dot{q}^{\alpha}, C_{\alpha}^{\prime(L, M)}(q)\right\}\left\langle\left|J_{(L, M)}\right|\right\rangle+\ldots,
$$

which will be specified later.

After substitution of the ansatz (13) into the model Lagrangian density (1) and integration over spatial coordinates, the Lagrangian has the form

$$
\begin{aligned}
L= & \frac{1}{2} \dot{q}^{\alpha} g_{\alpha \beta}(q, F) \dot{q}^{\beta}+a^{0} \frac{1}{2}\left\{\dot{q}^{\alpha}, C_{\alpha}^{\prime(2,0)}(q)\right\} \\
& +\left[(\dot{q})^{0}-\text { order terms }\right]
\end{aligned}
$$

where the metric tensor is

$$
\begin{aligned}
& g_{\alpha \beta}(q, F)= \\
& C_{\alpha}^{(L, M)}(q) E_{(L, M)\left(L^{\prime}, M^{\prime}\right)}(F) C_{\beta}^{\left(L^{\prime}, M^{\prime}\right)}(q),
\end{aligned}
$$

with the intermediate function $E_{(L, M)\left(L^{\prime}, M^{\prime}\right)}$ defined as

$$
E_{(L, M)\left(L^{\prime}, M^{\prime}\right)}(F)=-(-1)^{M} a_{L, M}(F) \delta_{L, L^{\prime}} \delta_{M,-M^{\prime}} .
$$

Note that the exact expression of the coefficient $a^{0}$ is not important for the calculation of $g_{\alpha \beta}$. There are five different quantum moments of inertia in (19):

$$
\begin{aligned}
& a_{(1,0)}(F)=\frac{1}{\mathrm{e}^{3} f_{\pi}} \times \\
& \int \mathrm{d}^{3} \tilde{r} \tilde{r}^{2} \sin ^{2} F\left(n_{0}^{2}-1\right)\left(1+F^{2}+\frac{\mathcal{I}}{r^{2}} \sin ^{2} F\right), \\
& a_{(1,1)}(F)=a_{(1,-1)}(F)=\frac{1}{2 \mathrm{e}^{3} f_{\pi}} \times \\
& \int \mathrm{d}^{3} \tilde{r} \tilde{r}^{2} \sin ^{2} F\left(n_{0}^{2}+1\right)\left(1+F^{2}+\frac{\mathcal{I}}{r^{2}} \sin ^{2} F\right),
\end{aligned}
$$




$$
\begin{aligned}
& a_{(2,0)}(F)=\frac{1}{\mathrm{e}^{3} f_{\pi}} \times \\
& \int \mathrm{d}^{3} \tilde{r} \tilde{r}^{2} \sin ^{2} F\left(n_{0}^{2}-1\right)\left\{\cos ^{2} F+n_{0}^{2} \sin ^{2} F\right. \\
& -\left(n_{0}^{2}-4 \cos ^{2} F+2 n_{0}^{2} \cos 2 F\right) F^{\prime 2} \\
& \left.+\frac{\mathcal{I}}{r^{2}} \sin ^{2} F\left[2 \cos ^{2} F+n_{0}^{2}(4-\cos 2 F)\right]\right\}, \\
& a_{(2,1)}(F)=a_{(2,-1)}(F)=\frac{1}{2 \mathrm{e}^{3} f_{\pi}} \times \\
& \int \mathrm{d}^{3} \tilde{r} \tilde{r}^{2} \sin ^{2} F\left\{3+2 \cos 2 F-3 n_{0}^{2}+4 n_{0}^{4} \sin ^{2} F\right. \\
& +\left[9+8 \cos 2 F-3 n_{0}^{2}-4 n_{0}^{4}(1+2 \cos 2 F)\right] F^{\prime 2} \\
& +\frac{\mathcal{I}}{r^{2}} \sin ^{2} F \times \\
& \left.\left[9+4 \cos 2 F-15 n_{0}^{2}+4 n_{0}^{4}(4-\cos 2 F)\right]\right\}, \\
& a_{(2,2)}(F)=a_{(2,-2)}(F)=\frac{1}{4 \mathrm{e}^{3} f_{\pi}} \times \\
& \int \mathrm{d}^{3} \tilde{r} \tilde{r}^{2} \sin ^{2} F\left\{-3-\cos 2 F-12 n_{0}^{2} \cos ^{2} F\right. \\
& +2 n_{0}^{4} \sin ^{2} F-2\left[3+2 \cos 2 F-24 n_{0}^{2} \cos ^{2} F\right. \\
& \left.+n_{0}^{4}(1+2 \cos 2 F)\right] F^{\prime 2}-\frac{2 \mathcal{I}}{r^{2}} \sin ^{2} F \times \\
& \left.\left[6+\cos 2 F-12 n_{0}^{2} \cos ^{2} F-n_{0}^{4}(4-\cos 2 F)\right]\right\},
\end{aligned}
$$

where the dimensionless variable $\tilde{r}=\mathrm{e} f_{\pi} r$ and $\mathrm{d}^{3} \tilde{r}=$ $\sin \theta \mathrm{d} \theta \mathrm{d} \varphi \mathrm{d} \tilde{r}$. These quantum moments depend on the chiral angle function $F(r)$, one component of the rational map vector $n_{0}$, and the function $\mathcal{I}(\theta, \varphi)$.

The canonical momenta are defined as

$$
p_{\beta}=\frac{\partial L}{\partial \dot{q}^{\beta}}=\frac{1}{2}\left\{\dot{q}^{\alpha}, g_{\alpha \beta}\right\}+a^{0} C_{\beta}^{\prime(2,0)}(q) .
$$

Note that the momenta do not commute and have terms which do not contain velocity. The parametrization $q^{\alpha}$ of the group manifold is significant for the definition of the canonical momenta. For the time being we do not require $\left[p_{\alpha}, p_{\beta}\right]=0$. The momenta and the conjugate coordinates satisfy the commutation relations $\left[p_{\beta}, q^{\alpha}\right]=-\mathrm{i} \delta_{\alpha \beta}$. This commutation relations determine the explicit expressions of the functions $f^{\alpha \beta}(q)$ in (14):

$$
\begin{aligned}
& f^{\alpha \beta}(q)=\left(g_{\alpha \beta}\right)^{-1}= \\
& C_{(L, M)}^{\prime \alpha}(q) E^{(L, M)\left(L^{\prime}, M^{\prime}\right)}(F) C_{\left(L^{\prime}, M^{\prime}\right)}^{\prime \beta}(q),
\end{aligned}
$$

where

$$
E^{(L, M)\left(L^{\prime}, M^{\prime}\right)}(F)=-\frac{(-1)^{M}}{a_{(L, M)}(F)} \delta_{L, L^{\prime}} \delta_{M,-M^{\prime}} .
$$

The functions $C_{(L, M)}^{\prime \alpha}(q)$ are defined in (A1.1), (A1.2).

It is possible to choose the parametrization on the SU(3) group manifold so that the eight operators

$$
\hat{R}_{(L, M)}=\frac{\mathrm{i}}{2}\left\{p_{\beta}, C_{(L, M)}^{\prime \beta}(q)\right\}
$$

are defined as the group generators satisfying the commutation relations (5). It is easy to check, that because of the choice (26) the requirement $\left[p_{\alpha}, p_{\beta}\right]=0$ is satisfied for certain. The proof for the $\mathrm{SU}(2)$ group can be found in [11]. The generators (26) act on the Wigner matrix of the $\mathrm{SU}(3)$ irreducible representation as right transformation generators:

$$
\begin{aligned}
& {\left[\hat{R}_{(L, M)}, D_{\left(\alpha_{1} L_{1} M_{1}\right)\left(\alpha_{2} L_{2} M_{2}\right)}^{(\lambda, \mu)}(q)=\right.} \\
& D_{\left(\alpha_{1} L_{1} M_{1}\right)\left(\alpha_{2} L_{2} M_{2}\right)}^{(\lambda, \mu) \times} \\
& \quad\left\langle\begin{array}{c}
(\lambda, \mu) \\
\left(\alpha_{2}^{\prime} L_{2}^{\prime} M_{2}^{\prime}\right)
\end{array}\left|J_{(L, M)}\right| \begin{array}{c}
(\lambda, \mu) \\
\left(\alpha_{2} L_{2} M_{2}\right)
\end{array}\right\rangle .
\end{aligned}
$$

The indices $\alpha_{1}$ and $\alpha_{2}$ label the multiplets of $(L, M)$. For instance, the left transformation generators are defined as

$$
\hat{L}_{(L, M)}=\frac{\mathrm{i}}{2}\left\{p_{\beta}, C_{(L, M)}^{\beta}(q)\right\} .
$$


Determination of functions $f^{\alpha \beta}(q)$ allows us to obtain an explicit expression of (18):

$$
\begin{aligned}
A^{\dagger} \dot{A}= & A^{\dagger}\left\{\dot{q}^{\alpha}, A\right\} \\
= & \frac{1}{2}\left\{\dot{q}^{\alpha}, C_{\alpha}^{\prime(L, M)}(q)\right\}\left\langle\left|J_{(L, M)}\right|\right\rangle \\
& -\frac{\mathrm{i}}{2} E^{\left(L_{1}, M_{1}\right)\left(L_{2}, M_{2}\right)}\left\langle\left|J_{\left(L_{1}, M_{1}\right)} J_{\left(L_{2}, M_{2}\right)}\right|\right\rangle \\
= & \frac{1}{2}\left\{\dot{q}^{\alpha}, C_{\alpha}^{\prime(L, M)}(q)\right\}\left\langle\left|J_{(L, M)}\right|\right\rangle \\
& +\frac{\mathrm{i}}{2 a_{0}} \cdot \mathbb{1}-\frac{\mathrm{i}}{2 a_{2}}\left\langle\left|J_{(2,0)}\right|\right\rangle,
\end{aligned}
$$

where $a_{0}$ and $a_{2}$ are constructed from the quantum moments of inertia:

$$
\begin{aligned}
& \frac{1}{a_{0}}=\frac{1}{3} \times \\
& \left(\frac{2}{a_{(1,0)}}+\frac{4}{a_{(1,1)}}+\frac{2}{a_{(2,0)}}+\frac{4}{a_{(2,1)}}+\frac{4}{a_{(2,2)}}\right) \\
& \frac{1}{a_{2}}=\frac{1}{\sqrt{3}} \times \\
& \left(-\frac{1}{a_{(1,0)}}+\frac{1}{a_{(1,1)}}+\frac{1}{a_{(2,0)}}+\frac{1}{a_{(2,1)}}-\frac{2}{a_{(2,2)}}\right) .
\end{aligned}
$$

The field (13) is substituted in the Lagrangian density (1) in order to obtain the explicit expression in terms of the collective coordinates and the space coordinates. After long calculation by using (29) and the commutation relation (15) (which is very important), we get a complete explicit expression of the Skyrme model Lagrangian density

$$
\begin{aligned}
& \mathcal{L}(q, \dot{q}, \varkappa)=\left\{\dot{q}^{\alpha}, C_{\alpha}^{\left(L, M_{1}\right)}(q)\right\}\left\{\dot{q}^{\beta}, C_{\beta}^{\left(L, M_{2}\right)}(q)\right\} \times \\
& \mathcal{V}_{1}(\varkappa)+\mathrm{i}\left\{\dot{q}^{\alpha}, C_{\alpha}^{\prime\left(L, M_{1}\right)}(q)\right\} \mathcal{V}_{2}(\varkappa)+\mathcal{V}_{3}(\varkappa)-\mathcal{M}_{\mathrm{cl}} .
\end{aligned}
$$

The function $\mathcal{V}_{1}$ in the first term results from the trace of two group generators (see (A3) below). The function $\mathcal{V}_{2}$ results from the trace containing three group generators (see (A4)). The function $\mathcal{V}_{3}$ results from the trace containing four group generators (A5). Expressions of $\mathcal{V}_{i}$ functions are presented in Appendix, see (A6)-(A8). The terms with functions $\mathcal{V}_{2}$ and $\mathcal{V}_{3}$ are absent in the semiclassical quantization.
Integration (31) over the space variables gives the Lagrangian

$$
\begin{aligned}
& L=\frac{1}{8}\left\{\dot{q}^{\alpha}, C_{\alpha}^{\prime\left(L_{1}, M_{1}\right)}(q)\right\} E_{\left(L_{1}, M_{1}\right)\left(L_{2}, M_{2}\right)} \times \\
& \left\{\dot{q}^{\beta}, C_{\beta}^{\left(L_{2}, M_{2}^{\prime}\right)}(q)\right\}+\mathrm{i}\left\{\dot{q}^{\alpha}, C_{\alpha}^{\prime(2,0)}(q)\right\} V_{2}+V_{3}-M_{\mathrm{cl}},
\end{aligned}
$$

where $V_{i}=\int \mathrm{d}^{3} x \mathcal{V}_{i}(\varkappa)$.

Sugano and collaborators [12] developed the qnumber variational method to formulate a theory that has the consistency between the Lagrangian and the Hamiltonian formalisms on the curved space of generalized coordinates. From (32) we specify the coefficient $a^{0}=2 \mathrm{i} V_{2}$ that has been undetermined in (23) and derive the Hamiltonian in a form

$$
\begin{aligned}
H= & \frac{1}{8}\left\{\dot{q}^{\alpha}, C_{\alpha}^{\prime\left(L_{1}, M_{1}\right)}(q)\right\} E_{\left(L_{1}, M_{1}\right)\left(L_{2}, M_{2}\right)} \times \\
& \left\{\dot{q}^{\beta}, C_{\beta}^{\left(L_{2}, M_{2}^{\prime}\right)}(q)\right\}-V_{3}+M_{\mathrm{cl}} \\
= & -\frac{1}{2} \hat{R}_{\left(L_{1}, M_{1}\right)} E^{\left(L_{1}, M_{1}\right)\left(L_{2}, M_{2}\right)} \hat{R}_{\left(L_{2}, M_{2}\right)} \\
& -\frac{2 V_{2}}{a_{(2,0)}} \hat{R}_{(2,0)}-2\left(\frac{V_{2}}{a_{(2,0)}}\right)^{2}-V_{3}+M_{\mathrm{cl}} .
\end{aligned}
$$

We define the state vectors as the complex conjugate Wigner matrix elements of the $(\Lambda, \Theta)$ representation depending on eight quantum variables $q^{\alpha}$ :

$$
\begin{aligned}
& \left|\begin{array}{c}
(\Lambda, \Theta) \\
\alpha, S, N ; \beta, S^{\prime}, N^{\prime}
\end{array}\right\rangle= \\
& \sqrt{\operatorname{dim}(\Lambda, \Theta)} D_{(\alpha, S, N)\left(\beta, S^{\prime}, N^{\prime}\right)}^{*(\Lambda, \Theta)}(q)|0\rangle .
\end{aligned}
$$

The indices $\alpha$ and $\beta$ label the multiplets of the $\operatorname{SO}(3)$ group. $|0\rangle$ denotes the vacuum state. Because of five different "moments of inertia", the vectors (34) are not the eigenstates of the Hamiltonian (34). The action of the Hamiltonian on vectors (34) following (27) can be expressed in terms of the "moments of inertia" $a_{(L, M)}$ and the SU(3) group Clebsch-Gordan coefficients.

We take into account the chiral symmetry breaking effects by introducing a term

$$
\mathcal{M}_{\mathrm{sb}}=\frac{1}{16} f_{\pi} m_{0}^{2} \operatorname{Tr}\left(U+U^{\dagger}-2\right),
$$

which takes an explicit form

$$
\mathcal{M}_{\mathrm{sb}}=\frac{1}{2} f_{\pi} m_{0}^{2} \sin ^{2} F,
$$


like in $B=1$ case [9].

\section{Conclusion}

We have considered a new rational map approximation ansatz for the Skyrme model which is the noncanonical embedded $\mathrm{SU}(3) \supset \mathrm{SO}(3)$ soliton with baryon number $B \geq 2$. The rational map ansatz is not spherically symmetric [13]. The canonical quantization leads to five different quantum "moments of inertia" in the Hamiltonian and the negative quantum mass corrections. The state vectors are defined as the SU(3) group representation $(\Lambda, \Theta)$ matrix depending on eight quantum variables $q^{i}$ because the ansatz does not commute with any generator of the group. The vectors (34) are not eigenvectors of the Hamiltonian for higher representations. The mixing is small. To find eigenstate vectors, the Hamiltonian matrix must be diagonalized in every $(\Lambda, \Theta)$ representation. If the baryon number $B=1$ and $\hat{n}=\hat{x}$, we get a soliton with two different "moments of inertia" which has been considered in [9].

\section{Appendix}

The functions $C_{(L, M)}^{\prime \alpha}(q)$ satisfy the following orthogonality relations:

$$
\begin{aligned}
& \sum_{L, M} C_{\alpha}^{\prime(L, M)}(q) \cdot C_{(L, M)}^{\prime \beta}(q)=\delta_{\alpha \beta} \\
& \sum_{\alpha} C_{\alpha}^{\prime(L, M)}(q) \cdot C_{\left(L^{\prime}, M\right)}^{\prime \alpha}(q)=\delta_{(L M)\left(L^{\prime} M^{\prime}\right)}
\end{aligned}
$$

The anticommutator of the canonical momenta and the functions $C_{(L, M)}^{\prime \alpha}(q)$ equals to

$$
\begin{gathered}
\frac{1}{2}\left\{\dot{q}^{\alpha}, C_{\alpha}^{\prime(L, M)}(q)\right\}=E^{(L, M)\left(L^{\prime}, M^{\prime}\right)} \frac{1}{2} \times \\
\left\{p_{\beta}, C_{\left(L^{\prime}, M^{\prime}\right)}^{\prime \beta}(q)\right\}-2 \mathrm{i} V_{2} E^{(2,0)(L, M)} .
\end{gathered}
$$

The trace of two group generators:

$$
\begin{gathered}
\operatorname{Tr}\left\langle\begin{array}{c}
(1,0) \\
D
\end{array}\left|J_{\left(L_{1}, M_{1}\right)} J_{\left(L_{2}, M_{2}\right)}\right| \begin{array}{c}
(1,0) \\
(D)
\end{array}\right\rangle= \\
(-1)^{M_{1}} 2 \delta_{L_{1}, L_{2}} \delta_{M_{1},-M_{2}} .
\end{gathered}
$$

The trace of three group generators:

$$
\begin{aligned}
& \operatorname{Tr}\left\langle\begin{array}{c}
(1,0) \\
D
\end{array}\left|J_{\left(L_{1}, M_{1}\right)} J_{\left(L_{2}, M_{2}\right)} J_{\left(L_{3}, M_{3}\right)}\right| \begin{array}{c}
(1,0) \\
(D)
\end{array}\right\rangle= \\
& (-1)^{M_{3}+1} 2 \sqrt{3}\left(\left[\begin{array}{ccc}
(1,1) & (1,1) & (1,1)_{\gamma=1} \\
\left(L_{1} M_{1}\right) & \left(L_{2} M_{2}\right) & \left(L_{3}-M_{3}\right)
\end{array}\right]\right. \\
& \left.+\left[\begin{array}{ccc}
(1,1) & (1,1) & (1,1)_{\gamma=2} \\
\left(L_{1} M_{1}\right) & \left(L_{2} M_{2}\right) & \left(L_{3}-M_{3}\right)
\end{array}\right] \frac{\sqrt{5}}{3}\right) .
\end{aligned}
$$

The trace of four group generators:

$$
\begin{aligned}
& \operatorname{Tr}\left\langle\begin{array}{c}
(1,0) \\
D
\end{array}\left|J_{\left(L_{1}, M_{1}\right)} J_{\left(L_{2}, M_{2}\right)} J_{\left(L_{3}, M_{3}\right)} J_{\left(L_{4}, M_{4}\right)}\right| \begin{array}{c}
(1,0) \\
(D)
\end{array}\right\rangle \\
&= 4\left[\left(2 L_{1}+1\right)\left(2 L_{2}+1\right)\left(2 L_{3}+1\right)\right]^{\frac{1}{2}} \times \\
& {\left[\left(2 L_{4}+1\right)\right]^{\frac{1}{2}} \sum_{k}(-1)^{u}\left\{\begin{array}{ccc}
L_{1} & L_{2} & k \\
1 & 1 & 1
\end{array}\right\}\left\{\begin{array}{ccc}
L_{3} & L_{4} & k \\
1 & 1 & 1
\end{array}\right\} \times } \\
& {\left[\begin{array}{ccc}
L_{1} & L_{2} & k \\
M_{1} & M_{2} & -u
\end{array}\right]\left[\begin{array}{ccc}
L_{3} & L_{4} & k \\
M_{3} & M_{4} & u
\end{array}\right] . }
\end{aligned}
$$

The terms included in the Lagrangian density (31):

$$
\begin{gathered}
\mathcal{V}_{1}(\varkappa)=\frac{f_{\pi}^{2}}{4}(-1)^{M_{1}}\left(D_{-M_{1}, M_{2}}^{L}(\varkappa)+\stackrel{+}{D}_{-M, M^{\prime}}^{L}(\varkappa)\right. \\
\left.-2 \delta_{-M_{1}, M_{2}}\right)+\frac{1}{16 \mathrm{e}^{2}}(-1)^{M^{\prime}} B_{m, m^{\prime}}(\varkappa) \times \\
\frac{3}{5-2 L}\left[\begin{array}{ccc}
L & 1 & L \\
M_{1}^{\prime} & m & M^{\prime}
\end{array}\right]\left[\begin{array}{ccc}
L & 1 & L \\
M_{2}^{\prime} & m^{\prime}-M^{\prime}
\end{array}\right] \times
\end{gathered}
$$

$$
\begin{aligned}
& \left(2 \delta_{M_{1}, M_{1}^{\prime}} D_{M_{2}, M_{2}^{\prime}}^{L}(\varkappa)-\delta_{M_{1}, M_{1}^{\prime}} \delta_{M_{2}, M_{2}^{\prime}}\right. \\
& \left.-D_{M_{1}, M_{1}^{\prime}}^{L}(\varkappa) D_{M_{2}, M_{2}^{\prime}}^{L}(\varkappa)\right),
\end{aligned}
$$




$$
\begin{aligned}
& \mathcal{V}_{2}(\varkappa)=\frac{f_{\pi}^{2}}{4}\left(\frac{(-1)^{M_{2}+M_{1}}}{\left.a_{\left(L, M_{2}\right.}\right)} \frac{\sqrt{2}}{\sqrt{3}} \sqrt{L^{2}+L+1} \times\right. \\
& {\left[\begin{array}{ccc}
L & L & 2 \\
M_{2}-M_{2}^{\prime} & M_{1}
\end{array}\right]\left(D_{M_{2}^{\prime}, M_{2}}^{L}(\varkappa)-\stackrel{+}{D_{M_{2}^{\prime}, M_{2}}^{L}}(\varkappa)\right)} \\
& \left.-\frac{1}{a_{2}}\left(D_{0, M_{1}}^{2}(\varkappa)-\stackrel{+}{D_{0, M_{1}}^{2}}(\varkappa)\right)\right) \\
& +\frac{1}{4 \mathrm{e}^{2}}(-1)^{M_{1}^{\prime}+M_{2}} \frac{\sqrt{2 \cdot 3}}{\left.a_{\left(L, M_{2}\right.}\right)} \frac{\sqrt{L^{2}+L+1}}{\sqrt{5-2 L}} \times \\
& B_{m, m^{\prime}}(\varkappa)\left[\begin{array}{ccc}
2 & 1 & 2 \\
M_{1}^{\prime \prime} & m & M_{1}^{\prime}
\end{array}\right]\left[\begin{array}{ccc}
L & 1 & L \\
M_{2}^{\prime \prime} & m^{\prime} & M_{2}^{\prime}
\end{array}\right] \times \\
& {\left[\begin{array}{ccc}
L & L & 2 \\
M_{2}^{\prime} & M_{2}^{\prime \prime \prime} & -M_{1}^{\prime}
\end{array}\right]\left(\delta_{M_{1} M_{1}^{\prime \prime}} \delta_{M_{2} M_{2}^{\prime \prime}} \stackrel{+}{D_{M_{2}^{\prime \prime \prime},-M_{2}^{\prime}}^{L}}(\varkappa)\right.} \\
& -\delta_{M_{1} M_{1}^{\prime \prime}} \delta_{M_{2} M_{2}^{\prime \prime}} \delta_{-M_{2} M_{2}^{\prime \prime \prime}}-\delta_{M_{1} M_{1}^{\prime \prime}} \delta_{-M_{2} M_{2}^{\prime \prime \prime}} \times \\
& \left.D_{M_{2}^{\prime \prime}, M_{2}}^{L}(\varkappa)+\delta_{M_{2} M_{2}^{\prime \prime}} \delta_{-M_{2} M_{2}^{\prime \prime \prime}} \stackrel{+}{D_{M_{1}^{\prime \prime}, M_{1}}^{2}}(\varkappa)\right),
\end{aligned}
$$

$\mathcal{V}_{3}(\varkappa)=\frac{f_{\pi}^{2}}{4} \frac{4\left(2 L_{1}+1\right)\left(2 L_{2}+1\right)}{a_{\left(L_{1}, M_{1}\right)} a_{\left(L_{2}, M_{2}\right)}}\left\{\begin{array}{ccc}L_{1} & L_{2} & k \\ 1 & 1 & 1\end{array}\right\}^{2} \times$

$$
\left[\begin{array}{lll}
L_{1} & L_{2} & k \\
M_{1} & M_{2} & u
\end{array}\right]^{2} D_{u, u}^{k}(\varkappa)+\frac{3}{a_{0}^{2}}+\frac{1}{a_{2}^{2}}\left(1+\stackrel{+}{D_{0,0}^{2}}(\varkappa)\right)
$$$$
-\frac{4}{a_{(L, M)}}\left(\frac{1}{a_{0}} D_{M, M}^{L}(\varkappa)-(-1)^{M} \frac{1}{a_{2}} \times\right.
$$$$
\left.\frac{\sqrt{L^{2}+L+1}}{\sqrt{2 \cdot 3}}\left[\begin{array}{ccc}
L & L & 2 \\
M & -M & 0
\end{array}\right] D_{M, M}^{L}(\varkappa)\right)
$$$$
-\frac{3}{2 \mathrm{e}^{2}} \frac{\left(2 L_{1}+1\right)\left(2 L_{2}+1\right)}{\sqrt{\left(5-2 L_{1}\right)\left(5-2 L_{2}\right)}}(-1)^{M_{1}+M_{2}+u} \times
$$$$
B_{m, m^{\prime}}(\varkappa)\left\{\begin{array}{ccc}
L_{1} & L_{1} & k \\
1 & 1 & 1
\end{array}\right\}\left\{\begin{array}{ccc}
L_{2} & L_{2} & k \\
1 & 1 & 1
\end{array}\right\}\left[\begin{array}{ccc}
L_{1} & 1 & L_{1} \\
M_{1}^{\prime} & m & M_{1}^{\prime \prime}
\end{array}\right] \times
$$$$
\left[\begin{array}{ccc}
L_{2} & 1 & L_{2} \\
M_{2}^{\prime} & m^{\prime} & M_{2}^{\prime \prime}
\end{array}\right]\left[\begin{array}{ccc}
L_{1} & L_{1} & k \\
M_{1} & -M_{1}^{\prime \prime} & u
\end{array}\right]\left[\begin{array}{ccc}
L_{2} & L_{2} & k \\
-M_{2} & M_{2}^{\prime \prime} & u
\end{array}\right] \times
$$

$$
\begin{aligned}
& \left(\frac { 1 } { a _ { ( L _ { 1 } , M _ { 1 } ) } a _ { ( L _ { 2 } , M _ { 2 } ) } } \left(\delta_{M_{2} M_{2}^{\prime}} D_{M_{1}^{\prime}, M_{1}}^{L_{1}}(\varkappa) \times\right.\right. \\
& \left(1-(-1)^{k}\right)-\delta_{M_{1} M_{1}^{\prime}} \delta_{M_{2} M_{2}^{\prime}}-D_{M_{1}^{\prime}, M_{1}}^{L_{1}}(\varkappa) \times \\
& \left.D_{M_{2}^{\prime}, M_{2}}^{L_{2}}(\varkappa)\right)+\frac{1}{a_{\left(L_{1}, M_{1}^{\prime}\right)} a_{\left(L_{2}, M_{2}\right)}} \delta_{M_{2} M_{2}^{\prime}} \times \\
& \left.D_{M_{1}^{\prime}, M_{1}}^{L_{1}}(\varkappa)\left(1+(-1)^{k}\right)\right),
\end{aligned}
$$

where $B_{m, m^{\prime}}(\varkappa)$ are

$$
\begin{aligned}
& B_{m, m^{\prime}}(\varkappa)=8(-1)^{m+m^{\prime}} \hat{n}_{-m} \hat{n}_{-m^{\prime}} \times \\
& \quad\left(\frac{1}{r^{2}} \mathcal{I} \sin ^{2} F-F^{\prime 2}\right)-(-1)^{m} \delta_{m,-m^{\prime}} \frac{8}{r^{2}} \mathcal{I} \sin ^{2} F .
\end{aligned}
$$

\section{References}

[1] T.H.R. Skyrme, A non-linear field theory, Proc. Roy. Soc. A 260, 127 (1961).

[2] C. Houghton, N. Manton, and P. Sutcliff, Rational maps, monopoles and skyrmions, Nucl. Phys. B 510, 507 (1998).

[3] A. Acus, J. Matuzas, E. Norvaišas, and D.O. Riska, The deuteron as a canonically quantized biskyrmion, Phys. Scripta 69, 260 (2004).

[4] O.V. Manko, N.S. Manton, and S.W. Wood, Light nuclei as quantized Skyrmions, Phys. Rev. C 76, 055203 (2007).

[5] G.S. Adkins, C.R. Nappi, and E. Witten, Static properties of nucleons in the Skyrme model, Nucl. Phys. B 228, 552 (1983).

[6] A. Acus, E. Norvaišas, and D.O. Riska, Stability and representation dependence of the quantum skyrmion, Phys. Rev. C 57, 2597 (1998).

[7] H. Walliser, The SU(n) Skyrme model, Nucl. Phys. A 548, 649 (1992).

[8] D. Jurčiukonis, E. Norvaišas, and D.O. Riska, Canonical quantization of SU(3) Skyrme model in a general representation, J. Math. Phys. 46, 072103 (2005).

[9] D. Jurčiukonis and E. Norvaišas, Quantum SU(3) Skyrme model with noncanonical embedded $\mathrm{SO}(3)$ soliton, J. Math. Phys. 48, 052101 (2007).

[10] A. Acus, E. Norvaišas, and D.O. Riska, The $\alpha$ particle as a canonically quantized multiskyrmion, Phys. Rev. C 74, 025203 (2006).

[11] K. Fujii, A. Kobushkin, K. Sato, and N. Toyota, Skyrme-model Lagrangian in quantum mechanics: SU(2) case, Phys. Rev. D 35, 1896 (1987). 
[12] T. Ohtani and R. Sugano, $Q$-number variational method for non-linear Lagrangian in quantum mechanics, Prog. Theor. Phys. 50, 1715 (1973).
[13] T. Ioannidou, B. Piette, P. Sutcliffe, and W. Zakrzewski, Skyrmions and rational maps, Nonlinearity 14, C1 (2001).

\title{
SO(3) RACIONALAUS ATVAIZDŽIO SOLITONAS KVANTINIAME SU(3) SKYRME'OS MODELYJE
}

\author{
D. Jurčiukonis, E. Norvaišas
}

VU Teorinès fizikos ir astronomijos institutas, Vilnius, Lietuva

\section{Santrauka}

Skyrme'os modelis su nekanoniškai įdètu $\mathrm{SU}(3) \supset \mathrm{SO}(3)$ solitonu nagrinėjamas racionalaus atvaizdžio artinyje, kai sprendinio topologinis krūvis $B \geq 2$. Didžiausias dèmesys skiriamas šio modelio grupiu teorijos aspektams. Pusiau klasikinis Skyrme'os modelio kvantavimo metodas remiasi prielaida, kad solitonas sukasi kaip kietas nekeičiantis formos kūnas. Straipsnyje naudojamas kanoninis kvantavimo metodas ab initio atsižvelgia ị reikalavimą, kad kolektyvinès koordinatès - kvantiniai kintamieji ir greičiai - neko- mutuoja. Tai keičia energijos funkcionalą, taigi ir variacinių sprendinių formą. Kvantiniai sprendiniai labai priklauso nuo klasikinio sprendinio, kurio aplinkoje kvantuojama. Pasirinktas racionalaus atvaizdžio artinys generuoja net penkis skirtingus Hamiltono operatoriaus ,inercijos momentus“. Aukštesniems SU(3) grupès įvaizdžiams gautas kvantinis Hamiltono operatorius nèra diagonalus nekanoninès bazès būsenų atžvilgiu. Šie aptarti sprendiniai gali būti panaudoti aprašyti lengvuosius atomo branduolius kaip specialius Skyrme'os modelio solitonus. 